OPEN ACCESS

Edited by:

Petar Bajic,

Cleveland Clinic, United States

Reviewed by:

Massimo Alfano,

San Raffaele Hospital (IRCCS), Italy

Alan J. Wolfe,

Loyola University Chicago,

United States

Viljemka Bučević Popović,

University of Split, Croatia

*Correspondence:

Peng Wu

doctorwupeng@gmail.com

Jie Zhao

zhaojie_0412@163.com

${ }^{t}$ These authors have contributed equally to this work

Specialty section:

This article was submitted to Microbiome in Health and Disease,

a section of the journal

Frontiers in Cellular

and Infection Microbiology

Received: 28 April 2020 Accepted: 16 November 2020

Published: 15 December 2020

Citation:

Zeng J, Zhang G, Chen C, Li K, Wen Y, Zhao J and Wu P (2020) Alterations in Urobiome in Patients With Bladder Cancer and Implications for Clinical Outcome: A Single-Institution Study.

Front. Cell. Infect. Microbiol. 10:555508. doi: 10.3389/fcimb.2020.555508

\section{Alterations in Urobiome in Patients With Bladder Cancer and Implications for Clinical Outcome: A Single-Institution Study}

\author{
Jiarong Zeng ${ }^{1,2 \dagger}$, Guihao Zhang ${ }^{1,2 \dagger}$, Chunxiao Chen ${ }^{1}$, Kun $L^{1}{ }^{1}$, Yuehui Wen ${ }^{1}$, Jie Zhao ${ }^{3 *}$ \\ and Peng $\mathrm{Wu}^{1,4^{*}}$ \begin{abstract}
People's Hospital (Huangtang Hospital), Meizhou, China, ${ }^{3}$ School of Pharmaceutical Sciences, Southern Medical University,
\end{abstract} \\ 'Department of Urology, Nanfang Hospital, Southern Medical University, Guangzhou, China, ${ }^{2}$ Department of Urology, Meizhou \\ Guangzhou, China, ${ }^{4}$ Clinical Microbiota Center, Nanfang Hospital, Southern Medical University, Guangzhou, China
}

Numerous studies indicate that resident microbiome exists in urine of healthy individuals and dysbiosis of the urobiome (urinary microbiome) may be associated with pathological conditions. This study was performed to characterize the alterations in urobiome and explore its implications of clinical outcome in male patients with bladder cancer. 62 male patients with bladder cancer and 19 non-neoplastic controls were recruited. The followup study cohort included 40 patients who were diagnosed with non-muscle invasive bladder cancer (NMIBC) and underwent transurethral resection of bladder tumor (TURBT). Mid-stream urine samples were collected from all the participants the day before cystoscopy. DNA was extracted from urine pellet samples and processed for high throughput 16S rRNA amplicon sequencing of the V4 region using Illumina MiSeq. Sequencing reads were filtered using QIIME and clustered using UPARSE. We found bacterial richness indices (Observed Species index, Chao1 index, Ace index; all $P<0.01$ ) increased in cancer group when compared with non-neoplastic group, while there were no differences in Shannon and Simpson index between two groups. During a median follow-up time of 12 (5.25-25) months, 5/40 (12.5\%)of the patients developed recurrence and no patient suffered from progression to muscle-invasive disease. Species diversity of the microbiome was significantly higher in the recurrence group compared with nonrecurrence group in patients with NMIBC after TURBT. The LEfSe analysis demonstrated that 9 genera were increased (e.g., Micrococcus and Brachybacterium) in recurrence group. To our knowledge we report the relative comprehensive study to date of the male bladder cancer urinary microbiome and its relationship to pathogenesis and clinical outcomes. Given our preliminary data, additional studies evaluating the urine microbiome in relation to clinical outcomes are warranted to improve our understanding of tumor recurrence after TURBT.

Keywords: microbiome, urinary tract, recurrence, diversity, bladder cancer, outcome 


\section{INTRODUCTION}

Bladder cancer is the 7th most commonly diagnosed cancer in males (Ferlay et al., 2013). In the past decades, bladder cancer has aroused scientists' attention for its high morbidity and recurrence rates. Unfortunately, the etiology and pathogenesis of bladder cancer is still ill-defined and clinical management remains challenging. Several important risk factors, including tobacco smoking, carcinogen exposure and genetic predisposition, may contribute to the pathogenesis of bladder cancer (Babjuk et al., 2019).

About $75 \%$ of bladder malignancies are diagnosed in superficial tumor stages (Babjuk et al., 2019). Superficial bladder cancer is characterized by high recurrence rate, with $50 \%-70 \%$ of patients whose bladders are not removed experiencing a relapse within 5 years of treatments (Ainsworth, 2017). The high variability in the recurrence rate indicates that the transurethral resection of bladder tumor (TURBT) was incomplete in a high percentage of patients. However, the factors that contribute to such enigmatic high recurrence rate are unknown. Because of costly lifetime surveillance with periodic cystoscopy and relevant recurrence rates, bladder cancer has the highest cost of any cancer when categorized on a per patient basis (Mossanen and Gore, 2014). This significant financial burden on the population and health care system calls for effective bladder cancer prevention and control (Fankhauser and Mostafid, 2018).

It is suggested that dysbiosis of the urobiome (urinary microbiome) may play an important role in the pathogenesis of numerous urologic diseases, such as overactive bladder and urgency urinary incontinence (Brubaker et al., 2014; Pearce et al., 2014; Pearce et al., 2015; Karstens et al., 2016; Thomas-White et al., 2016; Wu et al., 2017). Implications of urobiome in bladder cancer pathogenesis and therapeutics have also aroused abroad attention in the world (Ainsworth, 2017; Bajic et al., 2019; Markowski et al., 2019). It is reasonable to presume that dysbiosis of the urobiome may also plays a role in the pathogenesis of bladder cancer. Several lines of evidence underscored this hypothesis. $\mathrm{Xu}$ et al. reported some genera, such as Streptococcus, Pseudomonas and Anaerococcus, appearing more frequently in urothelial carcinoma patients (Xu et al., 2014). Adebayo and colleagues (2017) showed that some urinary taxa such as Fusobacterium and Sphingobacterium distinguished urogenital schistosomiasis-induced bladder pathologies from urogenital schistosomiasis infection alone and from healthy persons. It was reported that a higher abundance of Actinomyces was presented in bladder cancer patients, while Bifidobacterium, Streptococcus, Lactobacillus, and Veillonella were enriched in the control group (Bi et al., 2019). Liu et al. revealed higher relative abundances of the several microbial genera, such as Acinetobacter and Anoxybacillus, in the cancerous compared to the normal tissues (Liu et al., 2019). Our previous study (Wu et al., 2018) has shown increased bacterial richness, enrichment of some bacterial genera (e.g., Acinetobacter, Anaerococcus, and Sphingobacterium) and decrease of some bacterial genera (e.g., Serratia, Proteus, and Roseomonas) in bladder cancer group when compared with non- cancer group. It is worthy of note that higher abundance of the Acinetobacter in bladder cancer samples was also presented in several other studies (Liu et al., 2019; Mai et al., 2019). Acinetobacter components could play a role in carcinogenesis, by the activation of NF- $\mathrm{KB}$, in animal models of bladder cancer (Roperto et al., 2012). Further studies are needed to determine the role of Acinetobacter as a carcinogen or co-carcinogen in humans. Hourigan et al. analyzed different urinary collection methods (voided vs. cystoscopy) in patients with bladder cancer, and revealed a difference in beta diversity in males but not in females (Hourigan et al., 2020). Pederzoli et al. define a sexspecific common bladder cancer microbiome in tissue and urine, which confirms the concordance of voided urine and tissue microbiome (Pederzoli et al., 2020). They also found Klebsiella was more common in the urine of female patients versus healthy controls. Of note is that Klebsiella have been linked with bladder cancer in another study (Mansour et al., 2020). It is postulated that the Klebsiella could cause direct DNA-strand damage and generate genomic instability by releasing colibactin toxin (Kaur et al., 2018). Although data linking the urinary microbiome with clinical features of bladder cancer is increasing, to date, knowledge on the relationship between them is still limited.

We hypothesized that the dysbiosis of urobiome might play a role in the pathogenesis of bladder cancer and be associated with clinical outcomes. To investigate this, we prospectively collect mid-stream urine samples from male patients with bladder cancer the day before their cystoscopy, record their clinical outcome and perform integrated analysis. Attempt was made to explore the possible association of urinary microbiome with development and clinical outcome of bladder cancer, thus provides a basis for future research.

\section{MATERIALS AND METHODS}

\section{Study Population and Specimen Collection}

Only male participants were recruited in present study for two reasons: first, bladder cancer is male-dominated disease with almost 4.6 times as many as women (Ferlay et al., 2015); and second, there are significant gender differences of the urine microbiome (Kogan et al., 2015; Pederzoli et al., 2020). The total study cohort consisted of 62 bladder cancer, including 51 patients with non-muscle invasive bladder cancer (NMIBC) and 11 with muscle invasive bladder cancer (MIBC), and 19 nonneoplastic male patients between 2017 and 2019. All cancer cases were histologically confirmed as urothelial carcinoma and controls were cases without neoplastic conditions. All the participants were required to finish International Prostate Symptom Score (I-PSS) and those with moderate or severe symptoms (I-PSS $\geq 8$ ) were not eligible. To explore the role of the urinary microbiome composition in mediating clinical outcomes of patients with NMIBC after TURBT, we followed the participants after TURBT until the time to recurrence or end of follow-up. For the present study, the end of follow-up was set as September 30, 2019. Among 51 NMIBC, a total of 11 (21.6\%) patients received partial cystectomy or reject surgery; they were 
excluded from follow-up study. Finally, 40 patients with NMIBC at initial diagnosis who underwent TURBT in a single center between 2017 and 2019 were enrolled into follow-up study cohort.

We prospectively collected Mid-stream urine samples from male patients with bladder cancer the day before their cystoscopy. Patients' histopathological and clinical data as well as follow-up were recorded retrospectively after approval of the local ethics committee (NFEC-2020-045). All cases were reevaluated and classified according to the recent Tumor-NodeMetastasis Classification (TNM Classification) (2019) and the WHO 2016 grading classification of genitourinary tumors by experienced uropathologists. Subjects with a recent history of sexually transmitted infection, urinary tract infections, antibiotic usage for any indication (within 1 month) were not eligible. A structured questionnaire was finished by participants to collect information on socio-demographic characteristics. Data collection followed the principles outlined in the Declaration of Helsinki. Medicine Institutional Review Board of Southern Medical University approved this study and written informed consent was obtained from all participants. About $50 \mathrm{ml}$ of midstream urine specimens were collected by the clean catch method, and then centrifuged at $16,000 \mathrm{~g}$ for $10 \mathrm{~min}$ immediately. The pellets were stored at $-80^{\circ} \mathrm{C}$ until further processing.

\section{DNA Isolation and 16S rRNA Gene Sequencing}

In order to avoid contamination, DNA isolation was performed using the cultured cells protocol supplied with the DNeasy Blood \& Tissue Kit (Qiagen, Germany) in a laminar flow hood. The concentration of extracted DNA was determined through a Nanodrop ND-1000 spectrophotometer (Thermo Electron Corporation, USA). The genomic DNA isolated from the clinical samples was amplified using primer sets specific for V4 regions (515F: GTGCCAGCMGCCGCGGTAA; and 806R: GGACTACHVGGGTWTCTAAT). Extraction negative controls (no urine) and PCR negative controls (no template) were included to evaluate contribution of extraneous DNA from reagents. The resultant PCR products were purified by QIAquick PCR purification kit (Qiagen, Valencia, CA). Lastly, purified samples were normalized to equal DNA concentration and sequenced using the Illumina MiSeq sequencer (Illumina, Inc., USA). The raw sequence data reported in this paper have been deposited in the Genome Sequence Archive (Genomics, Proteomics \& Bioinformatics 2017) in National Genomics Data Center (Nucleic Acids Res 2020), Beijing Institute of Genomics (China National Center for Bioinformation), Chinese Academy of Sciences, under accession number CRA003441 that are publicly accessible at https://bigd.big.ac.cn/gsa.

\section{Bioinformatics Analysis}

To obtain clean reads, raw data were filtered to eliminate reads with adapter pollution and low quality by using QIIME (Version 1.80) (Caporaso et al., 2010). Filtered sequences were clustered by $97 \%$ identity into operational taxonomic units (OTUs) using UPARSE (Edgar, 2013), and subsequently, a single representative sequence from each clustered OTU was used to align to the SILVA database (Quast et al., 2013) by Ribosomal Database Project Classifier (Wang et al., 2007).

Alpha diversity, also called within-habitat diversity, was evaluated by using QIIME, which included the Observed Species index, Chaol index, Shannon index, Simpson index and Ace index. Among them, the Observed Species, Chaol and Ace index represent species richness, while Shannon and Simpson index are indicators of species diversity reflecting both species richness and evenness. The difference of alpha diversity between groups was measured by Wilcoxon RankSum Test (group number $=2$ ) and Kruskal-Wallis test $(n>2)$ using SPSS (version 22.0). Beta diversity, also called betweenhabitat diversity, was assessed to compare microbial composition by calculating the Bray Curtis, weighted UniFrac and unweighted UniFrac distances. Principal coordinate analysis (PCoA) was applied to generate three-dimensional plots in QIIME based on these distance matrices. The PERMANOVA was performed to test for statistical significance between groups using 999 permutations in QIIME. Differential abundance analysis between groups was performed using Metastats and $P$-values were adjusted for multiple hypotheses testing using the False Discovery Rate based on the Benjamini-Hochberg (White et al., 2009). To identify significantly different bacteria between groups, taxa summaries were reformatted and input into Linear discriminant analysis effect size (LEfSe) via the Huttenhower Lab Galaxy Server (Segata et al., 2011). The Kruskal-Wallis rank sum test and Wilcoxon test were used to identify biomarkers and linear discriminant analysis (LDA) was used to score them. Only taxa with logarithmic LDA score greater than 2 at a $P<0.05$ were considered significantly enriched. ROC (receiver operating characteristic) is a graphical plot which illustrates the performance of a binary classifier system as its discrimination threshold is varied. To explore the potential ability of the microbiome to identify bladder cancer status, ROC curve analysis was constructed to analyze the clinical accuracy of using the urinary microbiome for the diagnosis and prognostication of bladder cancer.

\section{Statistical Analysis}

Data are showed as median (first quartile to the third quartile) for continuous variables or number of cases (\%) for counts data. The statistical significance of differences between groups were measured using Mann-Whitney U-test for continuous variables and Pearson's chi-square test or Fisher' s Exact Test for count data through SPSS software (Version 22.0). All tests were based on two-tailed probability and $P$-value $<0.05$ were considered statistically significant. PASS program (PASS 11, NCSS, Kaysville UT, USA) provided estimates of power by simulation. Estimates were obtained 2000 simulations. Kaplan-Meier survival and a univariate Cox model were was assessed using $\mathrm{R}$ software to investigate the correlation between microbial diversity and recurrence-free survival. We estimated that with 62 patients in cancer group and 19 subjects in control group, we would have 
$90 \%$ power to detect differences at the 0.05 significance level (alpha) using a two-sided Mann-Whitney U-test.

\section{RESULTS}

\section{Subjects and Samples Characteristics}

A total of 99 participants were recruited, including 73 male cancer patients and 26 male non-neoplastic controls, while 11 cancer patients and seven controls were excluded for urine samples with too little sequencing reads. Therefore, the final study cohort consisted of 62 bladder cancer and 19 nonneoplastic controls (Table 1). Cancer group was composed of 51 patients with NMIBC and 11 patients with MIBC. No significant difference was observed in the demographic characteristics between cancer and non-cancer group, except cigarette smoking.

The follow-up study cohort consisted of 40 patients with NMIBC who underwent TURBT. The median (interquartile range $[\mathrm{IQR}])$ age was 65 (52.75-69.75 years) years and median (interquartile range [IQR]) follow-up for patients was 12 (5.2525 months) months (Table 2). No patient failed to follow up or fill out the surveys. After total follow-up, 5/40 (12.5\%) of the patients developed a recurrence on cystoscopy, but no patient suffered from progression to muscle-invasive disease. Smoking index was higher in recurrence (RE) patients when compared with non-recurrence (NR) patients.

\section{Sequencing Data, Alpha, and Beta Diversity}

A total of 9,947,140 clean reads were obtained from the 81 samples. The median number of reads in cancer patients was 115,990 , and in the non-cancer patients was 102,912 (Table 1, $P=0.002)$. The reads were classified into 3,031 OTUs used for downstream analysis. More OTUs were identified in urine from cancer patients, with an average of 165.13 OTUs per sample in cancer group and 85.74 OTUs per sample in control group $(P=0.0001)$.

The microbial richness indices (Observed Species index, Chaol index and Ace index; all $P<0.01$ ) were significantly higher in the cancer cohort than in the control cohort, while there were no differences in Shannon and Simpson index between two groups (Figures 1A-E, Table 1). PCoA revealed a clustering between OTUs from bladder cancer and control group by Bray-Curtis metric distances (Figure 1F), suggesting that the microbial communities exhibited phylogenetic closeness within each group $(P=0.001)$. By using ROC curve analysis, we found that AUC (area under the curve) was 0.79 (Observed Species index), 0.82 (Chaol index) and 0.84 (Ace index) (Figure 2). These AUC were all above 0.70 , meaning that species richness may have potential diagnostic value in patients with bladder cancer.

We then compared the microbial community of RE group and NR group. There were no differences in Observed Species index, Ace index and Chao index between RE group and NR group (Figures 3A-C). Higher Shannon index (diversity, $P<$ 0.05 ) and lower Simpson index (diversity, $P<0.05$ ) were represented in RE group compared with NR group (Figures 3D, E), suggesting that RE group have higher levels of diversity.

Based on these results, we then tested the relationship between microbial diversity and clinical outcome by stratifying the patients into two groups based on median diversity (Shannon and Simpson, 2.185 and 0.21, respectively). High alpha diversity group comprised patients with above median microbial diversity, while low alpha diversity those with below median diversity. Since Shannon and Simpson index both reflect the species diversity and Kaplan-Meier test showed a similar trend of the two indexes, only one chart made by Shannon was showed (Figure 3F). We found that patients with low alpha diversity had significantly prolonged recurrence-free survival than those with high alpha diversity using univariate Cox proportional hazard models $(P=0.019)$.

TABLE 1 | Comparisons of demographic characteristics and parameter of alpha diversity between cancer patients and non-cancer patients.

\begin{tabular}{|c|c|c|c|}
\hline & Cancer & Non-cancer & P-value \\
\hline \multicolumn{4}{|l|}{ DEMOGRAPHIC } \\
\hline Age & $56.00(45.0,65.3)$ & $46.00(35.0,64.0)$ & 0.07 \\
\hline $\mathrm{BMl}$ & 22.85(20.76,24.50) & 21.83(20.24,23.38) & 0.50 \\
\hline Smoking habit & $47(75.80)$ & $7(36.84)$ & $<0.01$ \\
\hline Smoking index & $550(0,800)$ & $\mathrm{O}(0,300)$ & $<0.01$ \\
\hline Drinking habit & $16(25.8)$ & $8(42.1)$ & 0.25 \\
\hline Hypertension & $20(32.2)$ & $4(21.1)$ & 0.40 \\
\hline Diabetes & $7(11.3)$ & $1(5.3)$ & 0.67 \\
\hline $\mathrm{CHD}$ & $3(4.8)$ & $2(10.5)$ & 0.33 \\
\hline $\mathrm{FHC}$ & $3(4.8)$ & $1(5.3)$ & 0.94 \\
\hline \multicolumn{4}{|l|}{ ALPHA DIVERSITY } \\
\hline Number of reads & $115990(103454,169,274.5)$ & 102912(61080,107378) & $<0.01$ \\
\hline Observed species & $147.50(101.25,206.25)$ & $57.0(52.00,115.00)$ & $<0.01$ \\
\hline Chao1 & $182.75(125.40,244.18)$ & $74.50(55.33,121.00)$ & $<0.01$ \\
\hline Ace & $191.54(132.24,248.03)$ & $86.01(57.51,120.66)$ & $<0.01$ \\
\hline Shannon & 2.49(1.63,3.10) & $2.25(1.79,2.82)$ & 0.42 \\
\hline Simpson & $0.185(0.10,0.32)$ & $0.23(0.13,0.32)$ & 0.45 \\
\hline
\end{tabular}

Data were presented as median (first quartile to the third quartile) for continuous variables or $n$ (\%) for counts. BMI, body mass index; CHD, coronary atherosclerotic heart disease; FHC, family history of cancer. 
TABLE 2 | Comparisons of clinical and histopathological parameters between Recurrence patients and non-Recurrence patients.

\begin{tabular}{|c|c|c|c|c|}
\hline Characteristic & ALL & Recurrence & Non-Recurrence & P-value \\
\hline Number of patients & 40 & 5 & 35 & - \\
\hline Median age (years) & $65.00(52.75,69.75)$ & $66.0(63.50,71.00)$ & $64.0(50.00,70.00)$ & 0.30 \\
\hline Smoking habit & $31(77.5 \%)$ & $5(100 \%)$ & $26(74.3 \%)$ & 0.57 \\
\hline Smoking index & $500.00(0.00,875.00)$ & $800.00(800.00,2000.00)$ & 400.00(0.00,800.00) & 0.21 \\
\hline \multicolumn{5}{|l|}{ Grading WHO2016 } \\
\hline PUNLMP & $10(25.0 \%)$ & $\mathrm{O}(0 \%)$ & $10(28.6 \%)$ & 0.30 \\
\hline Low grade & $16(40.0 \%)$ & $2(40 \%)$ & $14(40.0 \%)$ & \\
\hline High grade & $14(35.0 \%)$ & $3(60 \%)$ & $11(31.4 \%)$ & \\
\hline \multicolumn{5}{|l|}{ No. of tumors, n (\%) } \\
\hline Single & $34(85.0 \%)$ & $3(60 \%)$ & $31(88.6 \%)$ & 0.15 \\
\hline Multiple & $6(15.0 \%)$ & $2(40 \%)$ & $4(11.4 \%)$ & \\
\hline \multicolumn{5}{|l|}{ Stage, n (\%) } \\
\hline $\mathrm{Ta}$ & $29(72.5 \%)$ & $3(60 \%)$ & $26(74.3 \%)$ & 0.60 \\
\hline $\mathrm{T} 1$ & $11(27.5 \%)$ & $2(40 \%)$ & $9(25.7 \%)$ & \\
\hline \multicolumn{5}{|l|}{ Tumor diameter } \\
\hline$<3 \mathrm{~cm}$ & $31(77.5 \%)$ & $2(40 \%)$ & 29(82.9\%) & 0.07 \\
\hline$\geq 3 \mathrm{~cm}$ & $9(22.5 \%)$ & $3(60 \%)$ & $6(17.1 \%)$ & \\
\hline \multicolumn{5}{|c|}{ Regular Epirubicin Instillation therapy } \\
\hline YES & $27(67.5 \%)$ & $2(40 \%)$ & $25(71.4 \%)$ & 0.30 \\
\hline NO & $13(32.5 \%)$ & $3(60 \%)$ & $10(28.6 \%)$ & \\
\hline
\end{tabular}

Data were presented as median (first quartile to the third quartile) for continuous variables or $n$ (\%) for counts.

We next studied whether the microbial profile was associated with the recurrence of disease. The results showed that the microbiota composition of patients with recurrence was significantly different from that of patients without recurrence (PERMANOA, F=3.276, $P<0.01$, RE vs. NR, for unweighted UniFrac distances) (Figure 4A).

\section{LEfSe Analysis}

We identified the specific taxa associated with recurrence by using LEfSe analysis. The results showed that 9 genera were overrepresented in patients with recurrence, including Anoxybacillus, Massilia, Thermomonas, Brachybacterium, Micrococcus, Nocardioides, Larkinella, Jeotgalibacillus, and Geomicrobium (Figure 4B).
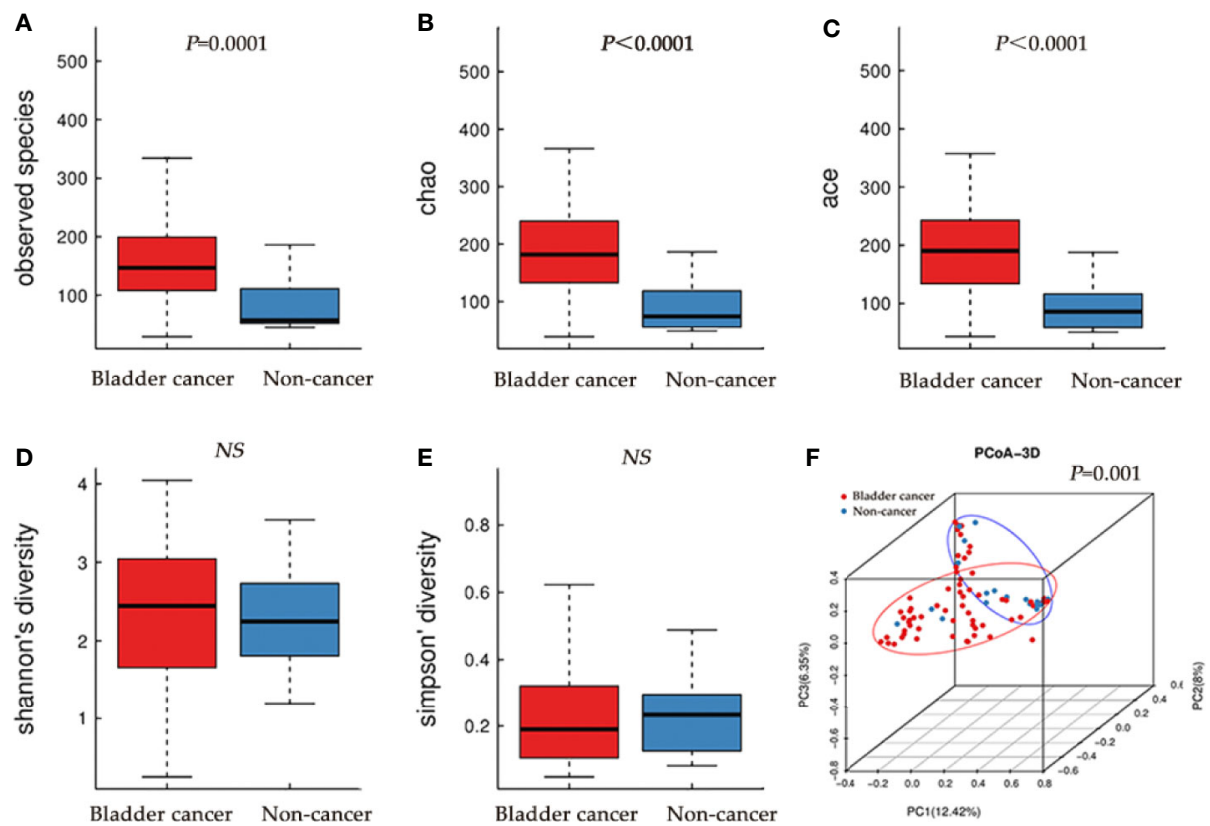

FIGURE 1 | Alpha and principal coordinate analysis (PCOA) for bladder-cancer samples and non-cancer samples. (A-E) Box-plot showing alpha diversity in samples using different metrics (A, observed species index; B, Chao index; C, Ace index; D, Shannon index; E, Simpson index). (F) PCoA plots of unweighed UniFrac distances in which samples were colored by clinical outcome. The PERMANOVA performed on the Bray-Curtis distances showed that the observed differences were statistically significant (999 permutations; $\mathrm{F}=2.71 ; \mathrm{P}=0.001$ ). 


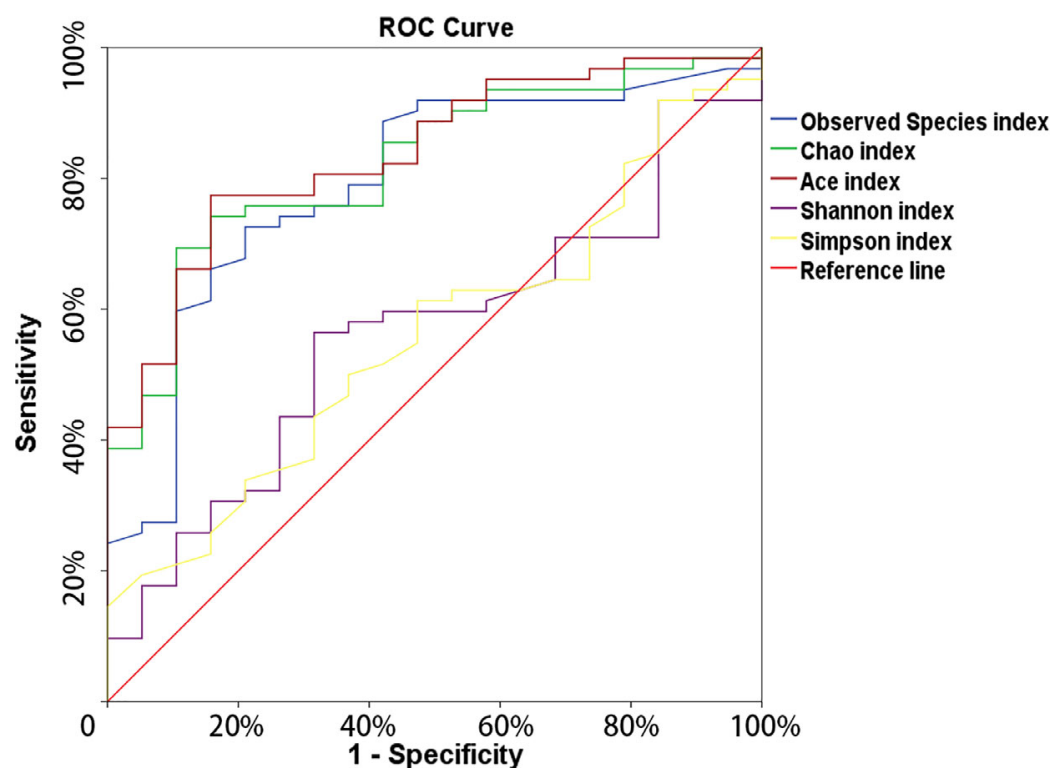

FIGURE 2 | The ROC curve used to analyze the clinical accuracy of using alpha- richness obtained from the bladder-cancer group and non-cancer group for the diagnosis of bladder cancer.

\section{Microbial Composition Analysis}

The most frequently detected phylum was Firmicutes (28.3\%RE, 37.7\%NR), followed by Proteobacteria (10.2\%RE, $25.8 \% \mathrm{NR}$ ) and Actinobacteria(6.7\%RE, 6.7\%NR) (Table 3). The microbial composition at class, order, family and genus level was demonstrated in Figures 5A-D and Table 3. The five most abundant class in RE group are Bacilli, Gammaproteobacteria, Actinobacteria, Bacteroidia and Clostridia. At the order level, Bacillales was the most predominant order in RE group, followed by Lactobacillales, Corynebacteriales, Bacteroidales,

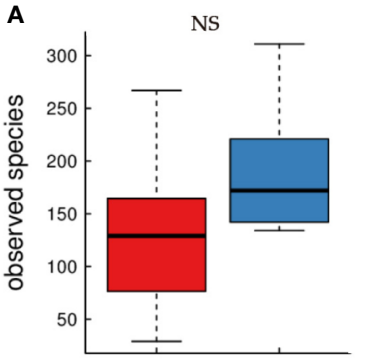

Non-recurrence Recurrence

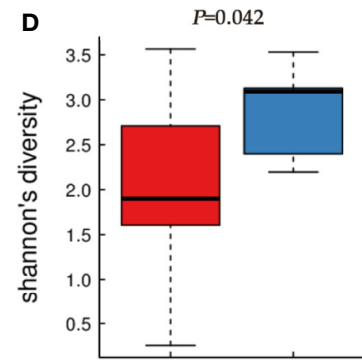

Non-recurrence Recurrence

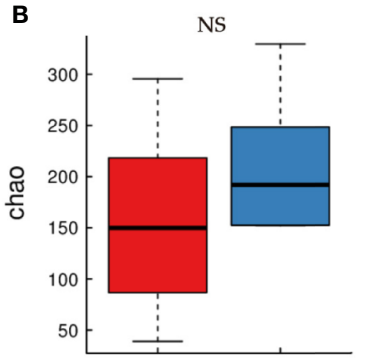

Non-recurrence Recurrence

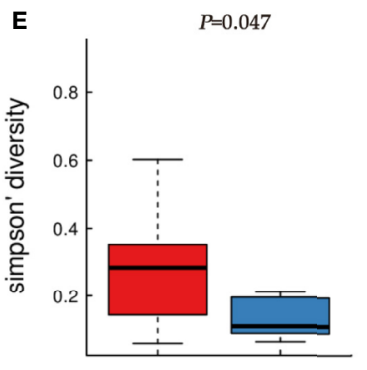

Non-recurrence Recurrence

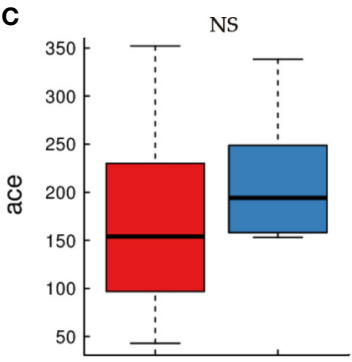

Non-recurrence Recurrence

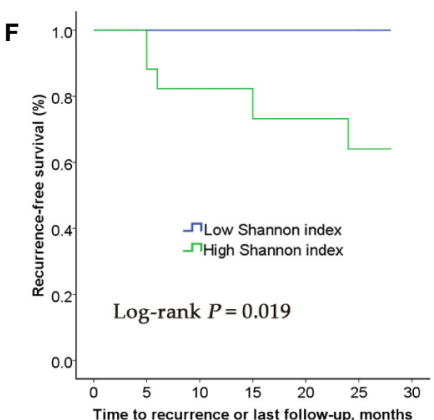

FIGURE 3 | Alpha diversity and Kaplan-Meier analysis for RE patients and NR patients urinary microbiome. Observed species (A); Chao index (B); Ace index (C); Shannon index (D); Simpson index (E). (F) Kaplan-Meier plot of patients with NMIBC defined by alpha diversity. 


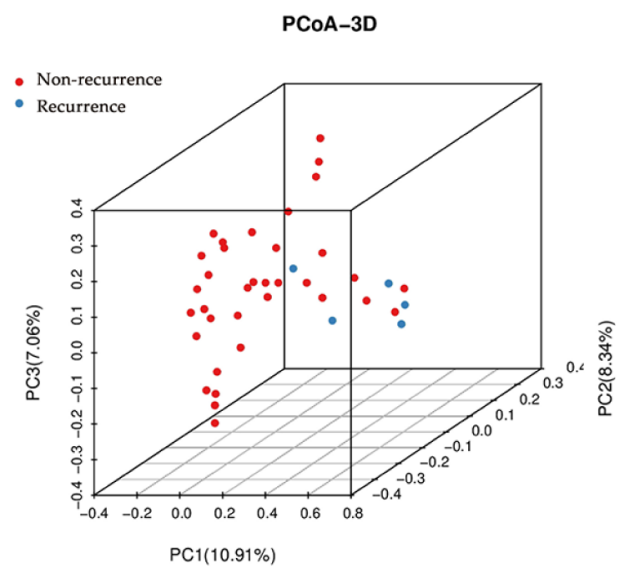

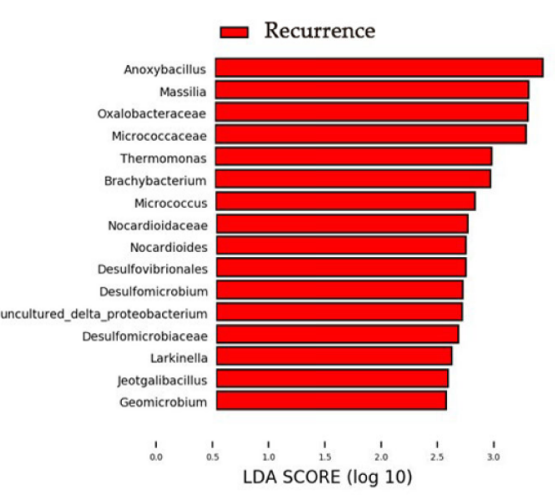

FIGURE 4 | Principal coordinate analysis and LEfSe analyses for RE patients and NR patients urinary microbiome. (A) Principal coordinate analysis plot of the urinary microbiome based on the unweighted distance metrics. (B) LEfSe analyses of urinary microbiomes of RE patients compared with NR patients. Genera enriched for RE group in red. No genera enriched for NR group according to LEfSe analyses. Only genera meeting a linear discriminant analysis score threshold $>2.5$ are shown.

Pseudomonadales, and Enterobacteriales. The most predominant family in RE group was Staphylococcaceae, followed by Streptococcaceae, Corynebacteriaceae, and Prevotellaceae. At the genus level, Staphylococcus was the most predominant genus in RE group, followed by Streptococcus, Prevotella, and Corynebacterium_1. By using Metastats algorithm after adjusting the False Discovery Rate, no significant difference was found of relative abundance between RE and NR group at class, order, family or genus level (Table 3 ). Of note, the relative abundance of Lactobacillus was higher in NR group than that in RE group (5.4\% vs. $0.04 \%$ ), although the difference was no statistically significant.

TABLE 3 | Comparison of relative abundance of urinary microbiome between NR group and RE group at all taxonomic levels.

\begin{tabular}{|c|c|c|c|c|c|}
\hline Taxa & & NR & RE & p-value & FDR \\
\hline \multirow[t]{4}{*}{ Phylum } & Firmicutes & 37.67 & 28.26 & 0.59 & 1.00 \\
\hline & Proteobacteria & 25.75 & 10.23 & 0.02 & 0.53 \\
\hline & Actinobacteria & 6.69 & 6.69 & 0.95 & 1.00 \\
\hline & Bacteroidetes & 6.37 & 5.81 & 0.89 & 1.00 \\
\hline \multirow[t]{5}{*}{ Class } & Bacilli & 28.67 & 25.39 & 0.89 & 1.00 \\
\hline & Gammaproteobacteria & 20.13 & 7.34 & 0.01 & 0.53 \\
\hline & Actinobacteria & 5.79 & 6.45 & 0.93 & 1.00 \\
\hline & Bacteroidia & 5.78 & 5.18 & 0.94 & 1.00 \\
\hline & Clostridia & 3.19 & 2.29 & 0.83 & 1.00 \\
\hline \multirow[t]{6}{*}{ Order } & Bacillales & 8.87 & 14.17 & 0.78 & 1.00 \\
\hline & Lactobacillales & 19.72 & 11.19 & 0.31 & 0.83 \\
\hline & Corynebacteriales & 2.70 & 5.49 & 0.74 & 1.00 \\
\hline & Bacteroidales & 5.77 & 5.18 & 0.94 & 1.00 \\
\hline & Pseudomonadales & 6.70 & 2.64 & 0.09 & 0.63 \\
\hline & Enterobacteriales & 11.24 & 2.21 & 0.03 & 0.63 \\
\hline \multirow[t]{5}{*}{ Family } & Staphylococcaceae & 5.42 & 12.15 & 0.72 & 1.00 \\
\hline & Streptococcaceae & 10.39 & 9.05 & 0.94 & 1.00 \\
\hline & Corynebacteriaceae & 2.54 & 5.39 & 0.76 & 1.00 \\
\hline & Prevotellaceae & 4.82 & 5.08 & 0.97 & 1.00 \\
\hline & Lactobacillaceae & 5.60 & 0.04 & 0.64 & 0.99 \\
\hline \multirow[t]{5}{*}{ Genus } & Staphylococcus & 5.17 & 11.89 & 0.76 & 1.00 \\
\hline & Streptococcus & 10.31 & 8.74 & 0.94 & 1.00 \\
\hline & Prevotella & 4.13 & 4.96 & 0.95 & 1.00 \\
\hline & Corynebacterium_1 & 1.64 & 4.54 & 0.80 & 1.00 \\
\hline & Lactobacillus & 5.44 & 0.04 & 0.06 & 0.90 \\
\hline
\end{tabular}

Data were reported as mean percentage; FDR, P-value after false discovery rate adjustment. 

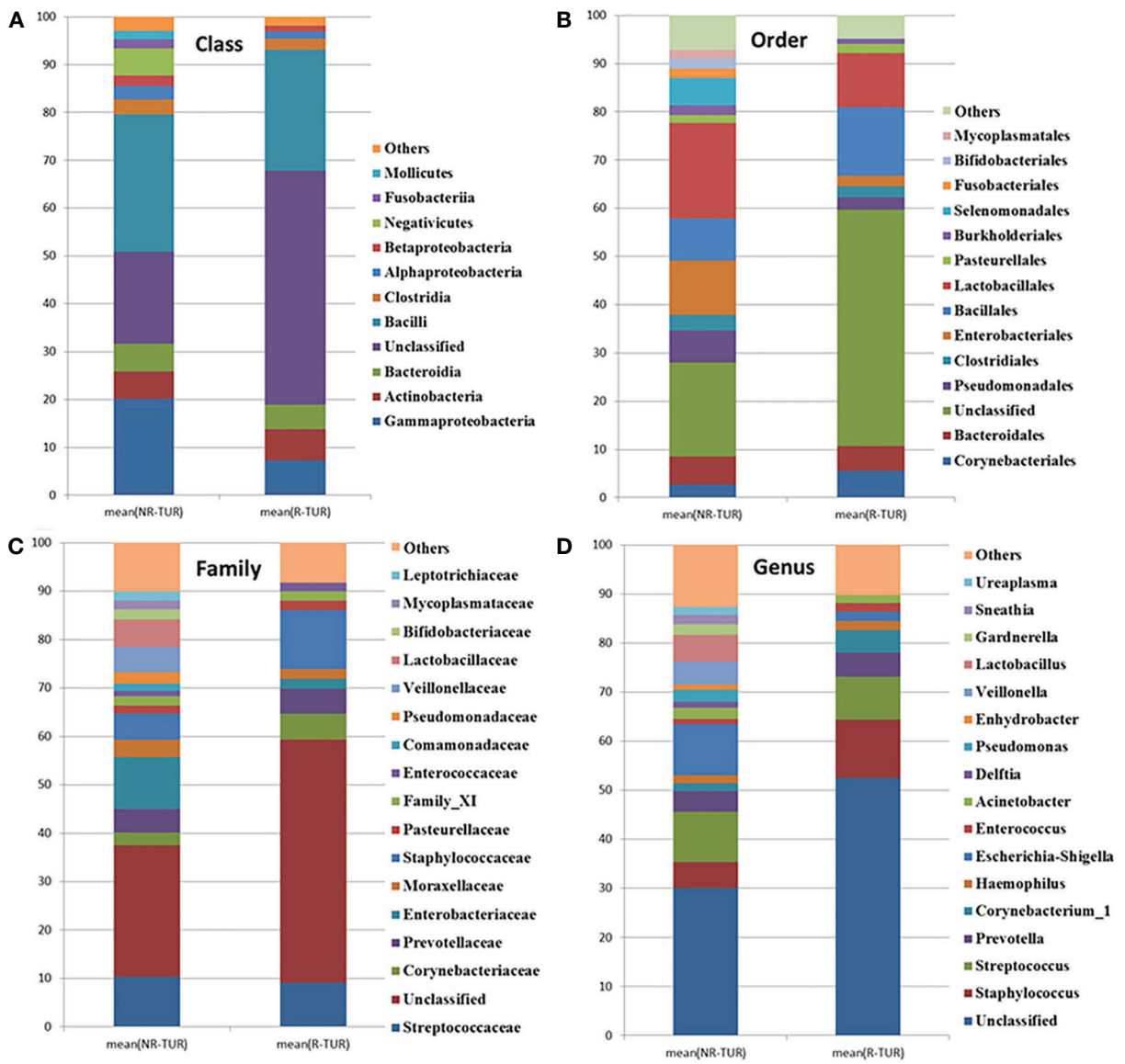

FIGURE 5 | The community barplot for RE patients and NR patients urinary microbiomes. Each colored box represents a bacterial taxon and each bar, a subject. The height of a colored box represents the relative abundance of that organism within the sample. Bacterial genera with a relative abundance $<1 \%$ are grouped as "Other". Percent of community abundance on Class (A), Order (B), Family (C), Genus (D) level.

\section{DISCUSSION}

The present evaluation conducted urinary microbial profiling of bladder cancer via 16S rRNA gene sequencing. In line with previous studies (Wu et al., 2018), we found that the Observed Species index, Chaol index and Ace index were significantly higher in cancer group when compared with non-cancer group, suggesting a higher degree of bacterial richness in cancer group. Further, we found that microbial richness might play a role in the diagnosis of bladder cancer by using ROC curve analysis.

Numerous studies have focused on the relationship between the host microbiome and cancer susceptibility or outcome in systems other than the urinary tract (Neto et al., 2015). For example, the intestinal microbiota may promote local inflammation and development of gastrointestinal cancers (Elinav et al., 2013; Garrett, 2015). Intestinal microbiome could also affect the outcomes of immunotherapeutic interventions in human cancers (Gopalakrishnan et al., 2018; Routy et al., 2018). Indirect effects of the microbiome on tumorigenesis are not well understood, and could include the modulation of antitumor immune responses (Schwabe and Jobin, 2013). Despite increasing evidence linking microbiota to cancer, the role of distal (gut) or local (urine) microbiota in urothelial carcinoma has not been fully illuminated. Urinary microbiome might create a barrier in uroepithelium, outcompete uropathogens, produce antimicrobial compounds and/or degrade harmful products (Whiteside et al., 2015).

This study makes a preliminary exploration of association of urinary microbiota and clinical feature of bladder cancer so as to promote better understanding between them. In present study, only male participants were recruited to avoid gender bias. Pederzoli et al. found distinct clustering of male versus female samples in both urine and bladder tissues, supporting the need for sex stratification in bladder cancer (Pederzoli et al., 2020). Sex related discrepancies in epidemiology, diagnosis, management, and outcomes among patients with bladder cancer have been reported (Bilski et al., 2020). It is reasonable to speculate that the difference of urinary pathogens may act an important role to gender diversities in these patients. The association of urine microbial richness with urinary health measures remains to be further explored. In the current study, we noted higher richness measures from males with bladder cancer versus those without cancer, which was in line with our previous study (Wu et al., 
2018). Higher richness in the bacterial community might not be a sign of a healthy microbiome, but likely suggested the overgrowth of various harmful bacteria or archaea in patients with bladder cancer. In the Patients with non-dialysis-dependent chronic kidney disease (CKD), the diversity measures (inverse Simpson index and Chao index) were higher than those from another study that evaluated midstream voided urine samples in women without CKD (Kramer et al., 2018). Among Patients with CKD, they also noted higher diversity measures from adults with urgency urinary incontinence (UUI) versus those without UUI. In another study, more diverse urinary microbiome was reported to correlates with less robust treatment response for female UUI with an anticholinergic treatment (Thomas-White et al., 2016). Women with more diverse microbiomes either required a higher dose of the anticholinergic for the same response or did not respond to the anticholinergic at all. However, information on midstream urine microbiome diversity remain very limited.

The hypothesis of microbiome serving as a non-invasive diagnosis tool for specific diseases or cancer, including type 2 diabetes (Qin et al., 2012), hepatocellular carcinoma (Ren et al., 2019), and colorectal cancer (Yu et al., 2017), has been proposed by several studies. Our study looked at urinary microbial alterations in bladder cancer in 62 patients and 19 healthy controls, endeavoring to assess the potential diagnostic value of urinary microbial. By using ROC curve analysis, we found the AUC of microbial richness index were all approximately $80 \%$, suggesting that microbial richness might play a potential role in the diagnosis of bladder cancer.

In the present study, we also explored the possible connection of urobiome and clinical outcomes of the NMIBC patients who underwent TURBT. Higher alpha diversity was observed in RE group when compared with NR group. Our results also demonstrated that the time to recurrence of bladder cancer differs between patients with higher alpha diversity and those with lower alpha diversity. Patients with higher alpha diversity tend to have a higher chance of recurrence compared with the men who had a lower diversity index.

Several studies have searched associations between the presence of microorganisms and clinical outcomes for specific diseases. R. Koedooder et al. (Koedooder et al., 2019) reported that vaginal microbiome profiling enables accurate prediction of both failure and success of fertility treatment. Women with an unfavorable profile had a seven times lower chance of achieving a pregnancy compared with the women who had a favorable vaginal microbiome profile. Erick Riquelme et al. (Riquelme et al., 2019) found that the tumor alpha diversity could serve as a predictor of survival outcome in resected pancreatic adenocarcinoma patients, suggesting the potential relevance of the microbiome composition in mediating pancreatic cancer progression. Urine microbial diversity may also relate to drug efficacy of patients with Urgency Urinary Incontinence (UUI) (Thomas-White et al., 2016). Compared with women with less diverse urine microbiomes, those with more diverse microbiomes either required a higher dose of the anticholinergic for the same response or did not respond to the anticholinergic at all (Thomas-White et al., 2016). These studies have preliminarily assessed the potential value of microbial composition in predicting clinical prognosis, while the specific mechanism is still unclear and it is necessary to be further verified by multicenter, prospective research.

Some bacterial taxa were richer in recurrence group by using the LEfSe analysis, such as Anoxybacillus and Micrococcus. Liu et al. also reported enrichment of Anoxybacillus in the cancerous tissues when compared with normal tissues (Liu et al., 2019). Micrococcus was recognized as an opportunistic pathogen and has been implicated in recurrent bacteremia, septic shock and endocarditis in immunosuppressed patients (Smith et al., 1999). However, their potential influence on contributing to cancer or vice versa is still unclear and more studies are necessary to determine the relationship between bladder cancer and described profiles.

Lower degree of the relative abundance of Lactobacillus was observed in RE group compared with NR group, although no statistically significant difference was found between two groups, which may be attributable to the low statistical power or variability within each group. The suppressor effect of Lactobacillus on superficial bladder cancer was an observed phenomenon but the specific mechanism is incompletely understood (Feyisetan et al., 2012). A team in Japan reported that bladder cancer patients who drank a probiotic containing Lactobacillus casei while also received chemotherapy treatments infused into the bladder had a $15 \%$ lower recurrence rate than those who received chemotherapy alone (Naito et al., 2008). Takahashi et al. showed that Lactobacillus casei intravesical therapy was superior to Bacille Calmette-Guerin (BCG) in reducing tumor growth in $\mathrm{C} 3 \mathrm{H}$ mice bearing $\mathrm{MBT}-2$ tumors (Takahashi et al., 2001). The mechanism of action by which lactobacilli appear to promote tumor resistance in humans has not been investigated but it has been implied that increased NK cell activity may play a role (Feyisetan et al., 2012).

To our knowledge, our study represents the first report to explore the influence of the urobiome on clinical outcomes in bladder cancer. The studies mentioned above preliminarily explored the associations between urobiome and clinical outcomes of NMIBC patients undergoing TURBT surgery, though the interaction is not clear. Our present study is also the relative comprehensive study in which profiling the urinary microbiome associated with bladder cancer to date. Nevertheless, there are several limitations as follows.

Firstly, the precision of our estimates is still limited by a moderate sample sizes and follow-up time. As such, we have now determined the sample sizes and follow-up time needed to further evaluate the relationships between microbiome and bladder cancer in our future larger studies. Secondly, we collect the samples only once and prior to the surgery in our study, so we cannot comment on microbial stability over time. Meanwhile, account must be taken for the outcomes of patients undergoing surgery depends on multiple clinical parameters (e.g. tumor factors, operation factors, bladder instillation regimen). Prospective studies are needed to disentangle the association between cancer development and microbial dysbiosis, as well as the possible role of these bacterial communities in the metabolism of carcinogenic compounds present in the urinary tract (Alfano et al., 2016). Moreover, the generalizability of predictive model is also needed to 
be proved in the external validation cohort. Last but not least, despite the use of voided samples avoids the participant burden of urinary catheterization, microbial assessments obtained using voided samples may include microbial contributions from adjacent pelvic niches (Kramer et al., 2018).

\section{CONCLUSION}

In conclusion, our study suggests that urobiome may be associated with bladder cancer and clinical outcomes of patients with NMIBC after TURBT. Given our preliminary data, we think that additional studies evaluating the urine microbiome in relation to clinical outcomes are warranted and hold promise to improve our understanding of disease recurrence after TURBT. The clinical applicability of these findings has not yet been integrated into daily practice. Developing a predictive test based on the urobiome composition will contribute to a personalized medicine approach in the future.

\section{DATA AVAILABILITY STATEMENT}

The datasets presented in this study can be found in online repositories. The names of the repository/repositories and accession number(s) can be found in the article/supplementary material.

\section{REFERENCES}

Adebayo, A. S., Suryavanshi, M. V., Bhute, S., Agunloye, A. M., Isokpehi, R. D., Anumudu, C. I., et al. (2017). The microbiome in urogenital schistosomiasis and induced bladder pathologies. PloS Negl. Trop. Dis. 11 (8), e5826. doi: 10.1371/journal.pntd.0005826

Ainsworth, C. (2017). Microbiome: A bag of surprises. Nature 551 (7679), S40S41. doi: 10.1038/551S40a

Alfano, M., Canducci, F., Nebuloni, M., Clementi, M., Montorsi, F., and Salonia, A. (2016). The interplay of extracellular matrix and microbiome in urothelial bladder cancer. Nat. Rev. Urol. 13 (2), 77-90. doi: 10.1038/nrurol.2015.292

Babjuk, M., Burger, M., Comperat, E. M., Gontero, P., Mostafid, A. H., Palou, J., et al. (2019). European Association of Urology Guidelines on Non-muscleinvasive Bladder Cancer (TaT1 and Carcinoma In Situ) - 2019 Update. Eur. Urol. 76 (5), 639-657. doi: 10.1016/j.eururo.2019.08.016

Bajic, P., Wolfe, A. J., and Gupta, G. N. (2019). The Urinary Microbiome: Implications in Bladder Cancer Pathogenesis and Therapeutics. Urology 126, 10-15. doi: 10.1016/j.urology.2018.12.034

Bi, H., Tian, Y., Song, C., Li, J., Liu, T., Chen, Z., et al. (2019). Urinary microbiota a potential biomarker and therapeutic target for bladder cancer. J. Med. Microbiol. 68 (10), 1471-1478. doi: 10.1099/jmm.0.001058

Bilski, K., Dobruch, J., Kozikowski, M., Skrzypczyk, M. A., Oszczudlowski, M., and Ostrowski, J. (2020). Urobiome in Gender-Related Diversities of Bladder Cancer. Int. J. Mol. Sci. 21 (12), 4488. doi: 10.3390/ijms21124488

Brubaker, L., Nager, C. W., Richter, H. E., Visco, A., Nygaard, I., Barber, M. D., et al. (2014). Urinary bacteria in adult women with urgency urinary incontinence. Int. Urogynecol. J. 25 (9), 1179-1184. doi: 10.1007/s00192-013-2325-2

Caporaso, J. G., Kuczynski, J., Stombaugh, J., Bittinger, K., Bushman, F. D., Costello, E. K., et al. (2010). QIIME allows analysis of high-throughput community sequencing data. Nat. Methods 7 (5), 335-336. doi: 10.1038/nmeth.f.303

Edgar, R. C. (2013). UPARSE: highly accurate OTU sequences from microbial amplicon reads. Nat. Methods 10 (10), 996-998. doi: 10.1038/nmeth.2604

\section{ETHICS STATEMENT}

The studies involving human participants were reviewed and approved by Medicine Institutional Review Board of Southern Medical University. The patients/participants provided their written informed consent to participate in this study.

\section{AUTHOR CONTRIBUTIONS}

Conception and design: JZe, GZ, PW. Acquisition of data: JZe, GZ, CC, KL. Analysis and interpretation of data: JZe, JZh. Drafting of the manuscript: JZe, GZ. Critical revision of the manuscript for important intellectual content: PW, JZh, YW. Statistical analysis: PW, JZh. Obtaining funding: PW. Supervision: PW. All authors contributed to the article and approved the submitted version.

\section{FUNDING}

This study is supported by the Natural Science Foundation of Guangdong Province (grant no. 2018A030313148), the Natural Science Foundation of Guangdong Province (grant no. 2020A1515011339) and the National Natural Science Foundation of China (grant no. 81870522).
Elinav, E., Nowarski, R., Thaiss, C. A., Hu, B., Jin, C., and Flavell, R. A. (2013). Inflammation-induced cancer: crosstalk between tumours, immune cells and microorganisms. Nat. Rev. Cancer 13 (11), 759-771. doi: 10.1038/nrc3611

Fankhauser, C. D., and Mostafid, H. (2018). Prevention of bladder cancer incidence and recurrence: nutrition and lifestyle. Curr. Opin. Urol. 28 (1), 88-92. doi: 10.1097/MOU.0000000000000452

Ferlay, J., Steliarova-Foucher, E., Lortet-Tieulent, J., Rosso, S., Coebergh, J. W., Comber, H., et al. (2013). Cancer incidence and mortality patterns in Europe: estimates for 40 countries in 2012. Eur. J. Cancer 49 (6), 1374-1403. doi: 10.1016/j.ejca.2012.12.027

Ferlay, J., Soerjomataram, I., Dikshit, R., Eser, S., Mathers, C., Rebelo, M., et al. (2015). Cancer incidence and mortality worldwide: sources, methods and major patterns in GLOBOCAN 2012. Int. J. Cancer 136 (5), E359-E386. doi: $10.1002 /$ ijc. 29210

Feyisetan, O., Tracey, C., and Hellawell, G. O. (2012). Probiotics, dendritic cells and bladder cancer. BJU Int. 109 (11), 1594-1597. doi: 10.1111/j.1464-410X.2011.10749.x

Garrett, W. S. (2015). Cancer and the microbiota. Science 348 (6230), 80-86. doi: $10.1126 /$ science.aaa4972

Gopalakrishnan, V., Spencer, C. N., Nezi, L., Reuben, A., Andrews, M. C., Karpinets, T. V., et al. (2018). Gut microbiome modulates response to antiPD-1 immunotherapy in melanoma patients. Science 359 (6371), 97-103. doi: 10.1126/science.aan4236

Hourigan, S. K., Zhu, W., Wong, W. S. W., Clemency, N. C., Provenzano, M., Vilboux, T., et al. (2020). Studying the urine microbiome in superficial bladder cancer: samples obtained by midstream voiding versus cystoscopy. BMC Urol. 20 (1):5. doi: 10.1186/s12894-020-0576-Z

Karstens, L., Asquith, M., Davin, S., Stauffer, P., Fair, D., Gregory, W. T., et al. (2016). Does the Urinary Microbiome Play a Role in Urgency Urinary Incontinence and Its Severity? Front. Cell Infect. Microbiol. 6:78:78. doi: $10.3389 /$ fcimb. 2016.00078

Kaur, C. P., Vadivelu, J., and Chandramathi, S. (2018). Impact of Klebsiella pneumoniae in lower gastrointestinal tract diseases. J. Dig. Dis. 19 (5), 262-271. doi: $10.1111 / 1751-2980.12595$ 
Koedooder, R., Singer, M., Schoenmakers, S., Savelkoul, P., Morre, S. A., de Jonge, J. D., et al. (2019). The vaginal microbiome as a predictor for outcome of in vitro fertilization with or without intracytoplasmic sperm injection: a prospective study. Hum. Reprod. 34 (6), 1042-1054. doi: 10.1093/humrep/dez065

Kogan, M. I., Naboka, Y. L., Ibishev, K. S., Gudima, I. A., and Naber, K. G. (2015). Human urine is not sterile - shift of paradigm. Urol. Int. 94 (4), 445-452. doi: 10.1159/000369631

Kramer, H., Kuffel, G., Thomas-White, K., Wolfe, A. J., Vellanki, K., Leehey, D. J., et al. (2018). Diversity of the midstream urine microbiome in adults with chronic kidney disease. Int. Urol. Nephrol. 50 (6), 1123-1130. doi: 10.1007/s11255-018-1860-7

Liu, F., Liu, A., Lu, X., Zhang, Z., Xue, Y., Xu, J., et al. (2019). Dysbiosis signatures of the microbial profile in tissue from bladder cancer. Cancer Med. 8 (16), 6904-6914. doi: 10.1002/cam4.2419

Mai, G., Chen, L., Li, R., Liu, Q., Zhang, H., and Ma, Y. (2019). Common Core Bacterial Biomarkers of Bladder Cancer Based on Multiple Datasets. BioMed. Res. Int. 2019:4824909. doi: 10.1155/2019/4824909

Mansour, B., Monyok, A., Makra, N., Gajdacs, M., Vadnay, I., Ligeti, B., et al. (2020). Bladder cancer-related microbiota: examining differences in urine and tissue samples. Sci. Rep. 10 (1), 11042. doi: 10.1038/s41598-020-67443-2

Markowski, M. C., Boorjian, S. A., Burton, J. P., Hahn, N. M., Ingersoll, M. A., Maleki, V. S., et al. (2019). The Microbiome and Genitourinary Cancer: A Collaborative Review. Eur. Urol. 75 (4), 637-646. doi: 10.1016/j.eururo.2018.12.043

Mossanen, M., and Gore, J. L. (2014). The burden of bladder cancer care: direct and indirect costs. Curr. Opin. Urol. 24 (5), 487-491. doi: 10.1097/ MOU.0000000000000078

Naito, S., Koga, H., Yamaguchi, A., Fujimoto, N., Hasui, Y., Kuramoto, H., et al. (2008). Prevention of recurrence with epirubicin and lactobacillus casei after transurethral resection of bladder cancer. J. Urol. 179 (2), 485-490. doi: 10.1016/j.juro.2007.09.031

Neto, A. G., Bradshaw, A. D., and Pei, Z. (2015). Microbiome, a new dimension in cancer research. Ann. Transl. Med. 3 (16), 229. doi: 10.3978/j.issn.23055839.2015.08.07

Pearce, M. M., Hilt, E. E., Rosenfeld, A. B., Zilliox, M. J., Thomas-White, K., Fok, C., et al. (2014). The female urinary microbiome: a comparison of women with and without urgency urinary incontinence. mBio 5 (4), e1214-e1283. doi: 10.1128/ mBio.01283-14

Pearce, M. M., Zilliox, M. J., Rosenfeld, A. B., Thomas-White, K. J., Richter, H. E., Nager, C. W., et al. (2015). The female urinary microbiome in urgency urinary incontinence. Am. J. Obstet. Gynecol. 213 (3), 341-347. doi: 10.1016/ j.ajog.2015.07.009

Pederzoli, F., Ferrarese, R., Amato, V., Locatelli, I., Alchera, E., Luciano, R., et al. (2020). Sex-specific Alterations in the Urinary and Tissue Microbiome in Therapy-naive Urothelial Bladder Cancer Patients. Eur. Urol. Oncol. doi: 10.1016/j.euo.2020.04.002

Qin, J., Li, Y., Cai, Z., Li, S., Zhu, J., Zhang, F., et al. (2012). A metagenome-wide association study of gut microbiota in type 2 diabetes. Nature 490 (7418), 5560. doi: $10.1038 /$ nature 11450

Quast, C., Pruesse, E., Yilmaz, P., Gerken, J., Schweer, T., Yarza, P., et al. (2013). The SILVA ribosomal RNA gene database project: improved data processing and web-based tools. Nucleic Acids Res. 41 (Database issue), D590-D596. doi: 10.1093/nar/gks1219

Ren, Z., Li, A., Jiang, J., Zhou, L., Yu, Z., Lu, H., et al. (2019). Gut microbiome analysis as a tool towards targeted non-invasive biomarkers for early hepatocellular carcinoma. Gut 68 (6), 1014-1023. doi: 10.1136/gutjnl-2017-315084

Riquelme, E., Zhang, Y., Zhang, L., Montiel, M., Zoltan, M., Dong, W., et al. (2019). Tumor Microbiome Diversity and Composition Influence Pancreatic Cancer Outcomes. Cell 178 (4), 795-806. doi: 10.1016/j.cell.2019.07.008
Roperto, S., Di Guardo, G., Leonardi, L., Pagnini, U., Manco, E., Paciello, O., et al. (2012). Bacterial isolates from the urine of cattle affected by urothelial tumors of the urinary bladder. Res. Vet. Sci. 93 (3), 1361-1366. doi: 10.1016/ j.rvsc.2012.06.009

Routy, B., Le Chatelier, E., Derosa, L., Duong, C., Alou, M. T., Daillere, R., et al. (2018). Gut microbiome influences efficacy of PD-1-based immunotherapy against epithelial tumors. Science 359 (6371), 91-97. doi: 10.1126/ science.aan 3706

Schwabe, R. F., and Jobin, C. (2013). The microbiome and cancer. Nat. Rev. Cancer 13 (11), 800-812. doi: 10.1038/nrc3610

Segata, N., Izard, J., Waldron, L., Gevers, D., Miropolsky, L., Garrett, W. S., et al. (2011). Metagenomic biomarker discovery and explanation. Genome Biol. 12 (6), R60. doi: 10.1186/gb-2011-12-6-r60

Smith, K. J., Neafie, R., Yeager, J., and Skelton, H. G. (1999). Micrococcus folliculitis in HIV-1 disease. Br. J. Dermatol. 141 (3), 558-561. doi: 10.1046/ j.1365-2133.1999.03060.x

Takahashi, T., Kushiro, A., Nomoto, K., Uchida, K., Morotomi, M., Yokokura, T., et al. (2001). Antitumor effects of the intravesical instillation of heat killed cells of the Lactobacillus casei strain Shirota on the murine orthotopic bladder tumor MBT-2. J. Urol. 166 (6), 2506-2511. doi: 10.1016/S0022-5347(05)65625-X

Thomas-White, K. J., Hilt, E. E., Fok, C., Pearce, M. M., Mueller, E. R., Kliethermes, S., et al. (2016). Incontinence medication response relates to the female urinary microbiota. Int. Urogynecol. J. 27 (5), 723-733. doi: 10.1007/ s00192-015-2847-x

Wang, Q., Garrity, G. M., Tiedje, J. M., and Cole, J. R. (2007). Naive Bayesian classifier for rapid assignment of rRNA sequences into the new bacterial taxonomy. Appl. Environ. Microbiol. 73 (16), 5261-5267. doi: 10.1128/AEM.00062-07

White, J. R., Nagarajan, N., and Pop, M. (2009). Statistical methods for detecting differentially abundant features in clinical metagenomic samples. PloS Comput. Biol. 5 (4), e1000352. doi: 10.1371/journal.pcbi.1000352

Whiteside, S. A., Razvi, H., Dave, S., Reid, G., and Burton, J. P. (2015). The microbiome of the urinary tract-a role beyond infection. Nat. Rev. Urol. 12 (2), 81-90. doi: 10.1038/nrurol.2014.361

Wu, P., Chen, Y., Zhao, J., Zhang, G., Chen, J., Wang, J., et al. (2017). Urinary Microbiome and Psychological Factors in Women with Overactive Bladder. Front. Cell Infect. Microbiol. 7:488:488. doi: 10.3389/fcimb.2017.00488

Wu, P., Zhang, G., Zhao, J., Chen, J., Chen, Y., Huang, W., et al. (2018). Profiling the Urinary Microbiota in Male Patients With Bladder Cancer in China. Front. Cell Infect. Microbiol. 8:167:167. doi: 10.3389/fcimb.2018.00167

Xu, W., Yang, L., Lee, P., Huang, W. C., Nossa, C., Ma, Y., et al. (2014). Minireview: perspective of the microbiome in the pathogenesis of urothelial carcinoma. Am. J. Clin. Exp. Urol. 2 (1), 57-61.

Yu, J., Feng, Q., Wong, S. H., Zhang, D., Liang, Q. Y., Qin, Y., et al. (2017). Metagenomic analysis of faecal microbiome as a tool towards targeted noninvasive biomarkers for colorectal cancer. Gut 66 (1), 70-78. doi: 10.1136/ gutjnl-2015-309800

Conflict of Interest: The authors declare that the research was conducted in the absence of any commercial or financial relationships that could be construed as a potential conflict of interest.

Copyright (c) 2020 Zeng, Zhang, Chen, Li, Wen, Zhao and Wu. This is an open-access article distributed under the terms of the Creative Commons Attribution License (CC BY). The use, distribution or reproduction in other forums is permitted, provided the original author(s) and the copyright owner(s) are credited and that the original publication in this journal is cited, in accordance with accepted academic practice. No use, distribution or reproduction is permitted which does not comply with these terms. 